\title{
High yield synthesis and structures of some achiral and chiral (diphosphine)tetracarbonylchromium(0) chelate complexes with tetracarbonyl(norbornadiene)chromium(0) as complexation reagent
}

\author{
Markus Strotmann, ${ }^{a}$ Rudolf Wartchow, ${ }^{b}$ and Holger Butenschön*a \\ ${ }^{a}$ Institut für Organische Chemie, Universität Hannover, Schneiderberg 1B, \\ D-30167 Hannover, Germany \\ ${ }^{b}$ Institut für Anorganische Chemie, Universität Hannover, Callinstraße 9, \\ D-30167 Hannover, Germany \\ E-mail: holger.butenschoen@mbox.oci.uni-hannover.de
}

\section{Dedicated to Professor Karsten Krohn on the occasion of his $60^{\text {th }}$ birthday} (received 19 Mar 04; accepted 15 Sept 04; published on the web 24 Sept 04)

\begin{abstract}
Tetracarbonyl(norbornadiene)chromium(0) was used as complexation reagent in the synthesis of tetracarbonylchromium chelate complexes of dppe, dppb, dppf, DIOP, BINAP, and Tol-BINAP. In all cases the reactions took place under mild reaction conditions with excellent, almost quantitative yields. X-ray crystal structure analyses of the dppf and BINAP tetracarbonylchromium( 0$)$ complexes are presented.
\end{abstract}

Keywords: Chromium complexes, norbornadiene, dppe, dppb, dppf, DIOP, BINAP, Tol-BINAP

\section{Introduction}

Diphosphanes are an important class of ligands in organometallic chemistry and have found wide applications, especially in catalytic reactions. ${ }^{1-3}$ In the last years chiral diphosphanes have been proven to be versatile tools for asymmetric metal catalyzed reactions. Most prominent examples in this context are atropisomeric chiral ligands such as 2,2'-bis(diphenylphosphino)-1,1'binaphthyl (BINAP) complexes, both enantiomers of the ligand are now commercially available. In connection with our interest in chiral complexation reagents we recently found that diphosphine tetracarbonychromium complexes can be obtained in very high, often quantitative yields by treatment of the ligands with tetracarbonyl(norbornadiene)chromium(0) (2). ${ }^{4,5}$ Here we report an improved synthesis of some known complexes of this kind, and in addition, high yield preparations of some new, chiral diphosphane tetracarbonylchromium complexes are presented. 
The nature of the complexation reagent favors the formation of monometallic chelate complexes under comparatively mild reaction conditions.

\section{Results and Discussion}

In 1961 Chatt and Watson observed the formation of chelate 3 in $71 \%$ yield when 1,2bis(diphenylphosphino)ethane (1) (dppe) was treated with hexacarbonylchromium. ${ }^{6}$ However, the reaction had to be carried out in a sealed tube at $150-160{ }^{\circ} \mathrm{C}$ at a pressure of 10 bar. ${ }^{6}$ Later, Werner and Prinz found that heating a mixture of dppe and hexacarbonylchromium under milder reaction conditions in boiling dioxane $\left(101^{\circ} \mathrm{C}\right)$ for $20 \mathrm{~h}$ gave the unchelated bimetallic complex in 55\% yield, some 3 has been obtained as a side product. ${ }^{7} \mathbf{3}$ has also been obtained by reaction of the ligand with $\mathrm{Cr}(\mathrm{CO})_{6}$ under microwave irradiation at $180{ }^{\circ} \mathrm{C}$ within $30 \mathrm{~s}$ in $86 \%$ yield. ${ }^{8}$ Using 2 instead of hexacarbonylchromium as the complexation reagent in $\mathrm{THF}$ at $50{ }^{\circ} \mathrm{C}$ gave an almost quantitative yield (99\%) after only 30 min reaction time (Scheme 1). Recently, a detailed FT-IR kinetic study of this reaction has been published independently. ${ }^{9}$

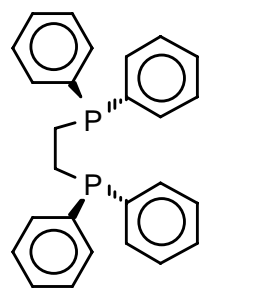

1

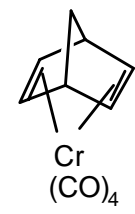

2
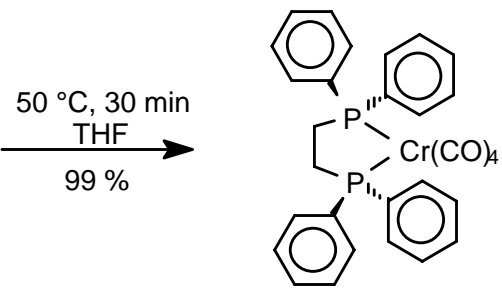

3

\section{Scheme 1}

Similarly, it was possible to coordinate 1,4-bis(diphenylphosphino)butane (dppb). Upon treatment with 2 in THF at $65^{\circ} \mathrm{C}$ for 60 min a 97\% yield of the seven-membered ring complex 4 was isolated (Figure 1). ${ }^{10,11}$

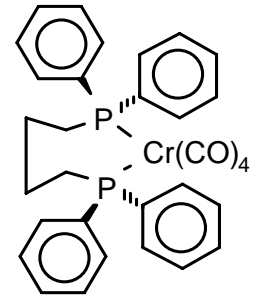

4

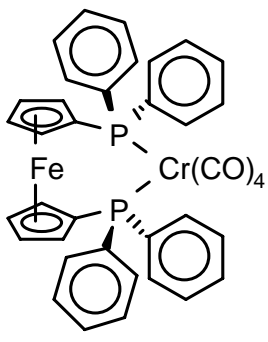

5

\section{Figure 1}


$1,1^{\prime}$-Bis(diphenylphosphino)ferrocene (dppf) is a bidendate ligand, ${ }^{12}$ which differs from the preceding examples in that the dppf ligand can adjust its ligand geometry by rotation of the cyclopentadienyl fragments in order to meet the steric requirements of a metal. Therefore, is was interesting to test the capability of (norbornadiene)tetracarbonylchromium (2) to react with dppf to give the corresponding tetracarbonylchromium complex 5 (Figure 1). Davison first prepared this complex in 1978 by treatment of dppf with hexacarbonylchromium in refluxing xylene in $73.1 \%$ yield. ${ }^{13}$ Later, Hor and Phang obtained 5 in addition to other, non chelated complexes by trimethylamine- $N$-oxide-inititated decarbonylation of hexacarbonylchromium in the presence of dppf in only $22 \%$ yield. ${ }^{14}$ The reaction of dppf with 2 in boiling THF gave a $98 \%$ yield of 5 as the only reaction product, which could be crystallized from heptane/benzene $(20: 1)$ at $5{ }^{\circ} \mathrm{C}$ to give crystals suitable for an X-ray crystal structure analysis (Figure 2).

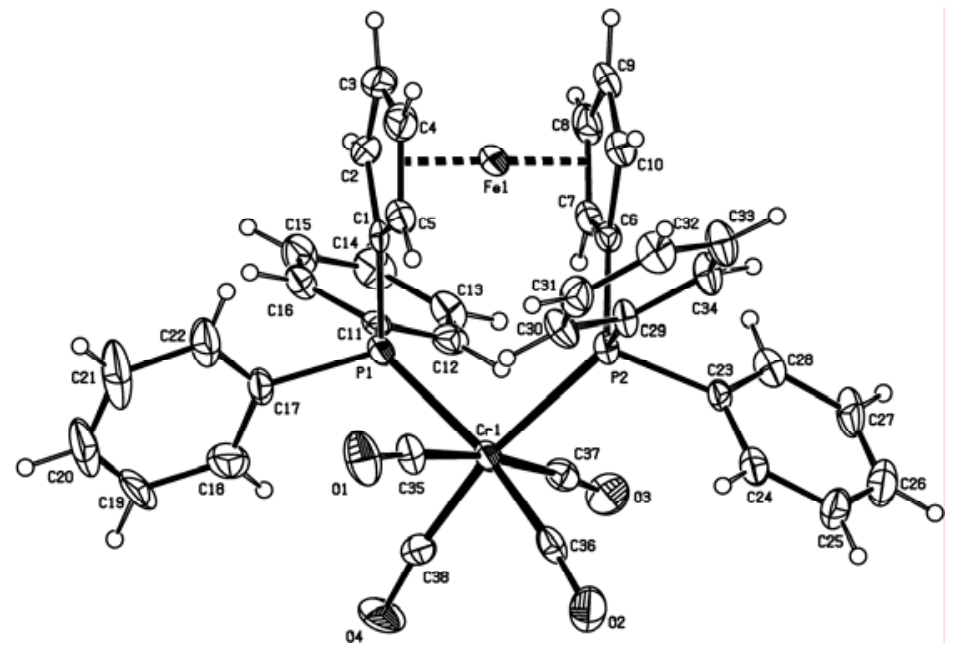

Figure 2. Structure of 5 in the crystal. Selected bond lengths $[\AA]$, bond angles $\left[^{\circ}\right]$, and torsion angles [ $\left.{ }^{\circ}\right]$ : Fe1-C1 2.022(4), Fe1-C2 2.032(4), Fe1-C3 2.048(5), Fe1-C4 2.049(5), Fe1-C5 2.032(5), Fe1-C6 2.029(4), Fe1-C7 2.024(5), Fe1-C8 2.054(5), Fe1-C9 2.053(5), Fe1-C10 2.029(4), Cr1-P1 2.4256(14), Cr1-P2 2.4739(14), P1-C1 1.819(5), P1-C11 1.826(5), P1-C17 1.866(5), P2-C6 1.796(5), P2-C23 1.853(5), P2-C29 1.813(5), P1-Cr1-P2 99.63(5), C1-P1-Cr1 116.2(2), C6-P2-Cr1 117.82(15), P2-Cr1-P1-C1 -28.8(2), P1-Cr1-P2-C6-19.5(2).

Complex 5 crystallizes in the monoclinic space group P 21/n with 8 molecules (4 pairs of enantiomers) in the unit cell. Presumably, crystal packing causes two slightly different molecular geometries. Chelation causes the cyclopentadienyl rings not to be completely parallel, they show a slight ring tilt of about $5^{\circ}$ thereby locating C- 1 and C- 6 a little bit closer to the iron atom than to the other cyclopentadienyl carbon atoms. The cyclopentadienyl rings are almost planar. The ferrocene conformation is characterized by a rotation of $15^{\circ}$ of the cyclopentadienyl rings, a value half way between the eclipsed $\left(0^{\circ}\right)$ and the staggered $\left(36^{\circ}\right)$ conformation. Compared to the 
known structure of the corresponding tetracarbonylmolybdenum complex, ${ }^{15}$ almost all differences can be explained by the smaller radius of the chromium atom.

In 1972 Kagan has introduced the historically important chiral diphosphane, (4S,5S)-[(2,2dimethyl-1,3-dioxolane-4,5-diyl)bis(methylene) $]$ diphenylphosphane $\left[(S, S)\right.$-DIOP, 6], ${ }^{16,17}$ which can be coordinated at $\mathrm{Cr}(\mathrm{CO})_{4}$ using $\mathbf{2}$ in boiling THF to give the seven-membered chelate 7 as a pale yellow, crystalline solid in excellent yield (99\%) (Scheme 2).

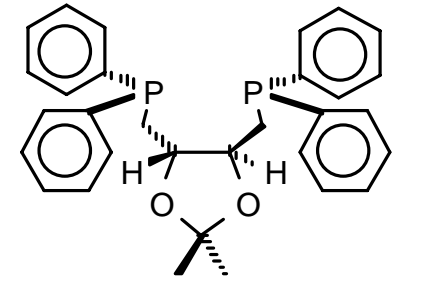

6

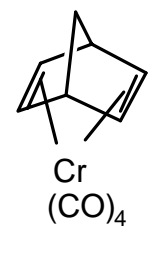

2
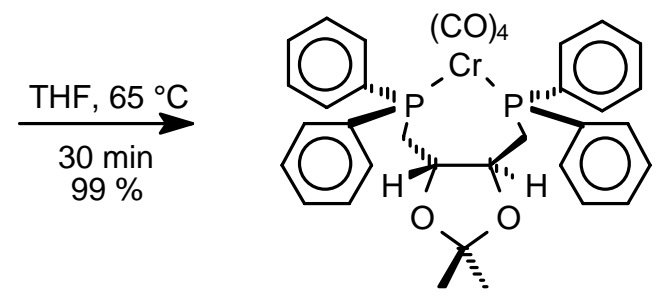

7

\section{Scheme 2}

2,2'-Bis(diphenylphosphino)-1,1'-binaphthyl (BINAP) (8a) is among the most prominent ligands used in asymmetric catalysis. ${ }^{18-20}$ The reaction of $(R)$-BINAP $(R-8 a)$ with 2 gave the $(R)$ BINAP tetracarbonylchromium complex $(R)-9 a$ in almost quantitative yield as a yellow crystalline material (Scheme 3), which rapidly decomposes when exposed to air. A short time after our first synthesis of this interesting complex Chauvin reported the observation of 9a by ${ }^{31} \mathrm{P}$ NMR and IR spectroscopy within a reaction sequence involving the treatment of methylated BINAP with $\mathrm{KHCr}(\mathrm{CO})_{5}{ }^{21}$

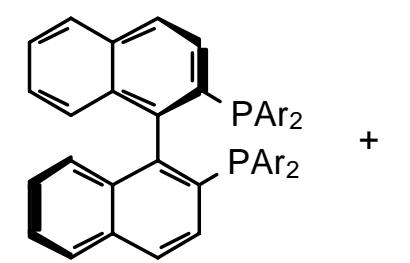

(R)-8a: $\mathrm{Ar}=\mathrm{Ph}$

$(R)-8 \mathbf{b}: \mathrm{Ar}=4-\mathrm{MeC}_{6} \mathrm{H}_{4}$

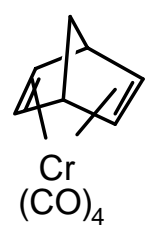

2

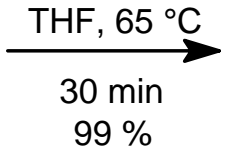

(R)-9a: $\mathrm{Ar}=\mathrm{Ph}(99 \%)$

(R)-9b : $\mathrm{Ar}=4-\mathrm{MeC}_{6} \mathrm{H}_{4}(99 \%)$

\section{Scheme 3}

Complex (R)-9a was dissolved with sonication in dibutyl ether at $40{ }^{\circ} \mathrm{C}$ and crystallized at $20{ }^{\circ} \mathrm{C}$ to give crystals suitable for an X-ray crystal structure analysis (Figure 3). (R)-9a crystallized in the orthorhombic space group P 21/21/21 with 4 molecules in the elemental cell solvated with one molecule of dibutyl ether (omitted for clarity). Presumably, due to crystal 
packing effects the structure does not show $C 2$ symmetry. The chromium atom displays a distorted octahedral coordination geometry, due to the P,P bridge all angles deviate by $1^{\circ}$ to $12^{\circ}$ from the ideal octahedral geometry. The axial CO ligands are bent away from the BINAP ligands by $23^{\circ}$. The average bond length of the Cr-P bonds $(2.415 \AA)$ is rather similar to the corresponding one in the dppf complex 5. The torsion angle between the planar naphthyl rings is about $77^{\circ}$. The comparison with the structure of the cationic rhodium $(R)$-BINAP norbornadiene perchlorate complex shows a deviation of the torsion by only $3^{\circ}$, although this complex has a square planar coordination geometry and a larger metal atom. ${ }^{19}$

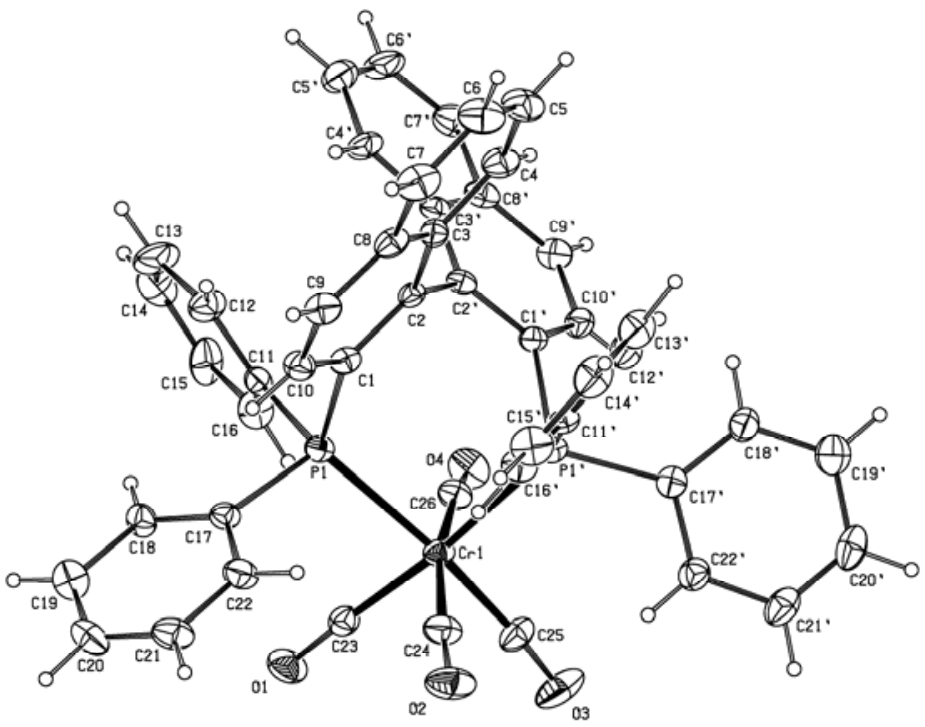

Figure 3. Structure of $(R)-9 \mathbf{a}$ in the crystal. Selected bond lengths $[\AA]$, bonding angles $\left[{ }^{\circ}\right]$, and dihedral angles $\left[^{\circ}\right]$ : Cr1-P1 2.4124(14), Cr1-P1' 2.4179(12), P1-C1 1.859(4), C1-C2 1.383(6), C1'-C2' 1.391(6), C2-C2' 1.494(6), Cr1-C23 1.854(4), Cr1-C24 1.886(6), Cr1-C25 1.826(5), Cr1-C26 1.869(6), C23-O1 1.149(4), C24-O2 1.138(6), C25-O3 1.155(5), C26-O4 1.151(5); P1Cr1-P1' 89.50(4), C1-P1-Cr1 119.5(2), C1'-P1'-Cr1 105.45(14), C1-C2-C2' 124.6(4), C1'-C2'-C2 121.9(5), C23-Cr1-P1 85.21(14), C24-Cr1-P1 99.2(2), C26-Cr1-P1 93.8(2), C24-Cr1-P1' 92.6(2), C25-Cr1-P1' 98.4(2); P1'-Cr1-P1-C1 -17.8(2), P1-Cr1-P1'-C1' -56.9(2), Cr1-P1-C1-C2 60.7(4), Cr1-P1'-C1'-C2' 78.0(3), P1-C1-C2-C2' 5.9(7), C10-C1-C2-C3 1.4(7), P1'-C1'-C2'-C2 15.3(5), C10'-C1'-C2'-C3' -0.3(6), C3-C2-C2'-C3' -75.7(5), C1-C2-C2'-C1'-77.7(6).

In some reactions 2,2'-bis(di-p-tolylphosphino)-1,1'-binaphthyl (Tol-BINAP, 8b) is preferred over BINAP, because Tol-BINAP is less easily oxidized. In an attempt to prepare the tetracarbonylchromium $(0)$ complex of $(R)-\mathbf{8 b}$ the ligand was treated with $\mathbf{2}$ in boiling THF for 30 min to give a $99 \%$ yield of the complex $(R)-9 \mathbf{b}$, which was obtained as a yellow, crystalline, airsensitive material (Scheme 3). 
In conclusion, we have shown that (norbornadiene)tetracarbonylchromium(0) (2) is an excellent reagent for the formation of diphosphane tetracarbonylchromium(0) complexes, which were obtained in very high, often quantitative yields under comparatively mild reaction conditions without the formation of non-chelated bimetallic complexes as side products. We currently investigate the chemical properties of these complexes in our laboratories.

\section{Experimental Section}

General Procedures. All operations were performed in flame-dried reaction vessels in an argon atmosphere using the Schlenk technique. Halogen-free solvents were distilled from sodiumpotassium alloy / benzophenone. Dichloromethane was distilled from calcium chloride. PE = petroleum ether, TBME = tert-butylmethyl ether. ${ }^{1} \mathrm{H}$ NMR: Bruker WP 80 (80 MHz), WP 200 SY (200.1 MHz), AM 400 (400.1 MHz). ${ }^{13}$ C NMR: Bruker WP 200 SY (50.3 MHz), AM 400 (100.1 MHz). Signal multiplicities were determined using APT and DEPT techniques. Chemical shifts refer to $\delta_{\mathrm{TMS}}=0$ or to residual solvent signals. ${ }^{22,23}{ }^{31} \mathrm{P}$ NMR: Bruker WP $200(81.3 \mathrm{MHz})$ and AM 400 (161.9 MHz), ${ }^{1} \mathrm{H}$ decoupling, external standard 85\% $\mathrm{H}_{3} \mathrm{PO}_{4}$. IR: Bruker ISS 25, Perkin-Elmer FT-IR 580 and 1710. MS: Finnigan MAT 112, 312 at $70 \mathrm{eV}$. FAB spectra were obtained using $o$-nitrobenzyl alcohol as the matrix and cesium as the ion source with Fisons VG Autospec. HRMS: Finnigan MAT 312, Fisons VG Autospec, peak matching with PFK. Combustion analyses: Heraeus CHN Rapid. Melting points were determined in sealed glass tubes with an apparatus according to Dr. Tottoli. Optical rotation: Perkin-Elmer polarimeter 241. Column chromatography: Silica gel (J. T. Baker, $40 \mu \mathrm{m}$ ) was degassed by heating with a heat gun at reduced pressure followed by setting under normal pressure with argon. This sequence was repeated five times. Separations were performed using flash chromatography. ${ }^{24}$

Preparation of diphosphane tetracarbonylchromium(0) chelate complexes. General procedure (GP). Diphosphane (1 equiv.) and tetracarbonyl(norbornadiene)chromium(0) (2) $)^{4,5}(1$ equiv.) in THF were stirred at the given temperature until 2 cannot be detected by TLC. The reaction progress is also indicated by a color change from orange-yellow to pale yellow or colorless. After removal of the solvent at reduced pressure the product is purified by column chromatography on silica gel.

Tetracarbonyl[1,2-bis(diphenylphosphino)ethane]chromium(0) (3). ${ }^{6}$ GP, 1,2-bis(diphenylphosphino)ethane (dppe, 1; $466 \mathrm{mg}, 1.17 \mathrm{mmol}), 2$ (300 mg, $1.17 \mathrm{mmol}$ ) in THF (100 mL), at $50{ }^{\circ} \mathrm{C}, 30 \mathrm{~min}$. Column chromatography (length $30 \mathrm{~cm}, \varnothing 3 \mathrm{~cm}$; TBME/PE 1:10 to 1:2). Pale yellow crystals 3 (651 mg $1.16 \mathrm{mmol}, 99 \%)$; mp $211^{\circ} \mathrm{C}$. The spectroscopic data (IR, ${ }^{1} \mathrm{H}$ NMR, ${ }^{13} \mathrm{C}$ NMR, MS) of 3 are identical with those reported. ${ }^{25,26}$

Tetracarbonyl[1,4-bis(diphenylphosphino)butane $]$ chromium(0) (4). ${ }^{10} \mathrm{GP}, 1,4$-bis(diphenylphosphino)butane (dppb; $895 \mathrm{mg}, 2.10 \mathrm{mmol}), 2(533 \mathrm{mg}, 2.08 \mathrm{mmol})$ in THF (100 mL), heating 
at reflux, $60 \mathrm{~min}$. Column chromatography (length $35 \mathrm{~cm}, \varnothing 3.5 \mathrm{~cm}$; TBME/PE 1:10 to 1:5). Pale yellow crystals 4 (1187 mg, $2.01 \mathrm{mmol}, 97 \%)$; mp $156{ }^{\circ} \mathrm{C}$ (dec.). The spectroscopic data (IR, ${ }^{1} \mathrm{H}$ NMR, ${ }^{13} \mathrm{C}$ NMR, MS) of $\mathbf{4}$ are identical with those reported. ${ }^{10,11}$

Tetracarbonyl[1,1'-bis(diphenylphosphino)ferrocene]chromium(0) (5). ${ }^{13}$ GP, 1,1'-bis(diphenylphosphino)ferrocene ${ }^{27}$ (330 mg, $\left.0.60 \mathrm{mmol}\right)$, of $2(150 \mathrm{mg}, 0.59 \mathrm{mmol})$ in THF (50 mL), heating at reflux, $24 \mathrm{~h}$. Column chromatography (length $30 \mathrm{~cm}, \varnothing 2.5 \mathrm{~cm}$; diethyl ether). Golden crystals 5 (415 mg, $0.58 \mathrm{mmol}, 97 \%)$; mp $>210{ }^{\circ} \mathrm{C}$. The spectroscopic data (IR, ${ }^{1} \mathrm{H}$ NMR, ${ }^{13} \mathrm{C}$ NMR, MS) of 5 are identical with those reported. ${ }^{14,27}$

Crystal structure analysis of $5^{28} \mathrm{C}_{38} \mathrm{H}_{28} \mathrm{CrFeO}_{4} \mathrm{P}_{2}$, crystal size $0.56 \times 0.09 \times 0.12 \mathrm{~mm}, a=$ 9.340(1), $b=34.695(3), c=20.306(2) \AA, \alpha=90, \beta=92.46(1), \gamma=90^{\circ}, V=6574.1(11) \AA^{3}, d_{\text {ber. }}$. $=1.452 \mathrm{~g} \mathrm{~cm}^{-3}, \mu=9.1 \mathrm{~cm}^{-1}, \mathrm{~F}(000)=2944 \mathrm{e}, Z=8$, crystal system monoclinic, space group $P 2_{1} / n$ (Nr. 14), Stoe IPDS (Imaging Plate) diffractometer, MoK $\alpha, \lambda=0.71073 \AA, T=300 \mathrm{~K}$, $2 \theta_{\text {min }}=4.0^{\circ}, 2 \theta_{\max }=48.3^{\circ}, \Delta \Phi=0.8^{\circ}, 33072$ measured reflections $(-10: 9, \pm 39,-22: 23), 10281$ independent and 3548 observed reflections [I $>2 \sigma]$, completeness of data $98.9 \%, R(\mathrm{I})=0.130$, absorption correction: none, extinction correction: none, refinement: SHELXL-93, $N_{\text {ref }}=10281$, $N_{\text {par }}=829, R_{\mathrm{gt}}(\mathrm{F})=0.0316, R_{\mathrm{w}}\left(\mathrm{F}^{2}\right)=0.0607\left[\mathrm{w}=1 / \sigma^{2}\left(\mathrm{~F}_{0}{ }^{2}\right)\right], \mathrm{S}=0.50$, min. $/$ max. residual electron density $-0.30 / 0.27 \mathrm{e} \AA^{-3}$.

\section{Tetracarbonyl[(4S,5S)-[(2,2-dimethyl-1,3-dioxolan-4,5-diyl)bis[methylene(diphenylphos-}

phino)]]chromium(0) [(S,S)-7]. GP， (4S-trans)-[(2,2-dimethyl-1,3-dioxolan-4,5-diyl)bis[methylene(diphenylphosphine)] ${ }^{16}(S, S-D I O P, 6 ; 230 \mathrm{mg}, 0.46 \mathrm{mmol}), 2$ (120 mg, $\left.0.47 \mathrm{mmol}\right)$ in THF $(50 \mathrm{~mL})$, heating at reflux for $30 \mathrm{~min}$. Column chromatography (length $30 \mathrm{~cm}, \varnothing 2.5 \mathrm{~cm}$; TBME/PE 1:15 to 1:5). Pale yellow, crystals (S,S)-7 (303 mg, $0.46 \mathrm{mmol}, 99 \%) ; \mathrm{mp} 163{ }^{\circ} \mathrm{C}$ (dec.). $[\alpha]_{D}^{20}-170.1$ (c = 1, THF). IR (KBr): $\widetilde{v} 3056(\mathrm{w}), 2984(\mathrm{w}), 2932(\mathrm{w}), 2880(\mathrm{w}), 2004$ (s, CO), 1920 (s, CO), 1884 (s, CO), 1484 (w), 1432 (m), 1372 (w), 1244 (m), 1160 (m), 1092 (m), 1052 (m), $884(\mathrm{~m}), 816(\mathrm{~m}), 740(\mathrm{~m}), 692(\mathrm{~m}), 672(\mathrm{~m}), 644(\mathrm{~s}), 512(\mathrm{~m}) \mathrm{cm}^{-1} .{ }^{1} \mathrm{H}$ NMR (400.1 MHz, acetone- $\left.d_{6}\right): \delta 1.18(\mathrm{~s}, 6 \mathrm{H}, 4-\mathrm{H}), 2.60-3.15(\mathrm{~m}, 4 \mathrm{H}, 1-\mathrm{H}), 3.62-3.73(\mathrm{~m}, 2 \mathrm{H}, 2-\mathrm{H})$, 7.43-7.58 (m, 16H), 7.67-7.78 (m, 4H). ${ }^{13} \mathrm{C} \mathrm{NMR} \mathrm{(100.6} \mathrm{MHz,} \mathrm{acetone-d} d_{6}$, APT): $\delta 27.6(-, \mathrm{s}$, $\mathrm{C}-4), 35.5\left(+, \mathrm{t},{ }^{1} J_{\mathrm{C}, \mathrm{P}}=10 \mathrm{~Hz}, \mathrm{C}-1\right), 78.9\left(-, \mathrm{t},{ }^{2} J_{\mathrm{C}, \mathrm{P}}=8 \mathrm{~Hz}, \mathrm{C}-2\right), 109.2(+, \mathrm{s}, \mathrm{C}-3), 129.6[-, \mathrm{t}$, ${ }^{3} J_{\mathrm{C}, \mathrm{P}}=4 \mathrm{~Hz}, \mathrm{C}-7(9)$ or C-13(15)], $129.6\left[-, \mathrm{t},{ }^{3} J_{\mathrm{C}, \mathrm{P}}=4 \mathrm{~Hz}, \mathrm{C}-7(9)\right.$ or C-13(15)], $130.0(-, \mathrm{s}, \mathrm{C}-8$ or C-14), $131.0\left[-, \mathrm{t},{ }^{2} J_{\mathrm{C}, \mathrm{P}}=4 \mathrm{~Hz}\right.$ C-6(10) or C-12(16)], 131.3 (-, s, C-8 or C-14), 134.5 [-, t, ${ }^{2} J_{\mathrm{C}, \mathrm{P}}=6 \mathrm{~Hz}, \mathrm{C}-6(10)$ or C-12(16)], $136.4\left(+, \mathrm{t},{ }^{1} J_{\mathrm{C}, \mathrm{P}}=16 \mathrm{~Hz}, \mathrm{C}-5\right.$ or C-11), $142.3\left(+, \mathrm{t},{ }^{1} J_{\mathrm{C}, \mathrm{P}}=\right.$ $21 \mathrm{~Hz}, \mathrm{C}-5$ or C-11), $223.1\left(+, \mathrm{t},{ }^{2} J_{\mathrm{C}, \mathrm{P}}=13 \mathrm{~Hz} \mathrm{CO}_{\mathrm{ax}}\right), 226.2\left(+, \mathrm{t},{ }^{2} J_{\mathrm{C}, \mathrm{P}}=9 \mathrm{~Hz}, \mathrm{CO}_{\text {äq }}\right) .{ }^{31} \mathrm{P}\left\{{ }^{1} \mathrm{H}\right\}$ NMR (161.9 MHz, acetone- $\left.d_{6}\right): \delta 42.0$ (s). MS (70 eV, $\left.180{ }^{\circ} \mathrm{C}\right): \mathrm{m} / \mathrm{z}(\%) 663(2)[\mathrm{M}+1]^{+}, 662(3)$ $\left[\mathrm{M}^{+}\right], 578(2)[\mathrm{M}-3 \mathrm{CO}], 550$ (10) [M-4CO], 549 (52), 498 (1) [M-4CO-Cr], 274 (2), 155 (7), 135 (7), 122 (46), 121 (100), 99 (23), 91 (8), 77 (23), 73 (28), 59 (57), 52 (2) $\left[{ }^{52} \mathrm{Cr}^{+}\right]$. MS-FAB: $\mathrm{m} / \mathrm{z}(\%) 662(20)\left[\mathrm{M}^{+}\right], 550(100)$ [M-4CO]. HRMS $\left(\mathrm{C}_{35} \mathrm{H}_{32} \mathrm{CrO}_{6} \mathrm{P}_{2}\right)$ : Calcd. 662.1079; found 662.1074. Anal. Calcd for $\mathrm{C}_{35} \mathrm{H}_{32} \mathrm{CrO}_{6} \mathrm{P}_{2}$ (496.44): C, 63.45; H, 4.87. Found: C, 63.28; H, 4.88.

Tetracarbonyl[(R)-1,1'-binaphthalen-2,2'-diyl[bis(diphenylphosphino)]]chromium(0) [(R)9a]. GP, (R)-2,2'-bis(diphenylphosphino)-1,1'-binaphthyl $[(R)$-BINAP, $(R)-8 \mathbf{a} ; 414$ mg, 
$0.67 \mathrm{mmol}$ ] and $2(170 \mathrm{mg}, 0.66 \mathrm{mmol})$ in THF $(70 \mathrm{~mL})$, heating at reflux for $30 \mathrm{~min}$. Column chromatogrphy (length $30 \mathrm{~cm}, \varnothing 3 \mathrm{~cm}$ TBME 1:10 to 1:5). Pale yellow crystals (R)-9a (518 mg, $0.66 \mathrm{mmol}, 99 \%$ ); $\mathrm{mp} 181{ }^{\circ} \mathrm{C}$ (dec.). $[\alpha]_{D}^{20}+35.2$ (c=0.5, THF). IR (KBr): $\widetilde{v} 3052(\mathrm{w}), 2000(\mathrm{~s}$, CO), 1912 (s, CO), 1880 (s, CO), 1584 (w), 1500 (w), 1480 (w), 1432 (m), 1304 (w), 1088 (w), $816(\mathrm{w}), 744(\mathrm{~m}), 696(\mathrm{~m}), 676(\mathrm{~m}), 648(\mathrm{~m}), 524(\mathrm{~m}) \mathrm{cm}-1.1 \mathrm{H}-\mathrm{NMR}\left(400.1 \mathrm{MHz}\right.$, benzene- $\left.d_{6}\right)$ : $\delta$ 6.36-7.94 (m, 32H). 13C-NMR (100.6 MHz, benzene-d $d_{6}$ APT): $\delta 125.9$ (-, s), 126.4 (-, s), $127.2\left(-, \mathrm{t}, J_{\mathrm{C}, \mathrm{P}}=4.6 \mathrm{~Hz}\right), 127.9(-, \mathrm{m}), 128.1\left(-, \mathrm{t}, J_{\mathrm{C}, \mathrm{P}}=5.0 \mathrm{~Hz}\right), 128.2-128.5(-, \mathrm{m}), 129.7$ (-, s), $131.8(+, \mathrm{s}), 132.0\left(+, \mathrm{t}, J_{\mathrm{C}, \mathrm{P}}=1.0 \mathrm{~Hz}\right), 132.2(+, \mathrm{s}), 133.7(+, \mathrm{s}), 133.8\left(-, \mathrm{t}, J_{\mathrm{C}, \mathrm{P}}=6.2 \mathrm{~Hz}\right)$, $134.6\left(-, \mathrm{t}, J_{\mathrm{C}, \mathrm{P}}=5.4 \mathrm{~Hz}\right), 138.5\left(+, \mathrm{t}, J_{\mathrm{C}, \mathrm{P}}=7.0 \mathrm{~Hz}\right), 223.8\left(+, \mathrm{CO}_{\mathrm{ax}}, \mathrm{t},{ }_{2} J_{\mathrm{C}, \mathrm{P}}=13.1 \mathrm{~Hz}\right), 227.1(+$, $\mathrm{CO}$ äq, t, $\left.{ }_{2} J_{\mathrm{C}, \mathrm{P}}=8.2 \mathrm{~Hz}\right) .{ }_{31} \mathrm{P}\{1 \mathrm{H}\}-\mathrm{NMR}\left(161.9 \mathrm{MHz}\right.$, benzene- $\left.d_{6}\right): \delta 57.3(\mathrm{~s}) . \mathrm{MS}(70 \mathrm{eV}$, $\left.240{ }^{\circ} \mathrm{C}\right): \mathrm{m} / \mathrm{z}(\%) 731$ (1) [M-2CO], 676 (16), 675 (25) [M-4CO], 438 (36), 437 (100) [M-4CO$\mathrm{Cr}-\mathrm{PPh}_{2}$ ], 412 (5), 357 (4), 326 (4), 281 (9), 183 (6), 52 (6) [52 $\left.\mathrm{Cr}+\right]$. HRMS (C44H32 $\left.\mathrm{CrP}_{2}\right)$ : Calcd. 674.1384; found 674.1385. Anal. Calcd for $\mathrm{C}_{48} \mathrm{H}_{32} \mathrm{CrO}_{4} \mathrm{P}_{2}$ (786.72): C, 73.28; H, 4.10. Found: C, 73.18; H, 4.20 .

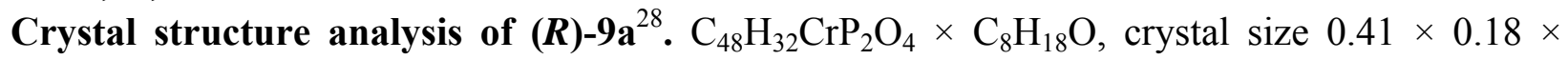
$0.14 \mathrm{~mm}, a=12.240(1), b=14.531(1), c=27.154(2) \AA, \alpha=90, \beta=90, \gamma=90^{\circ}, V=4829.6(6)$ $\AA^{3}, d_{\text {ber. }}=1.261 \mathrm{~g} \mathrm{~cm}^{-3}, \mu=3.5 \mathrm{~cm}^{-1}, \mathrm{~F}(000)=1920 \mathrm{e}, Z=4$, crystal system orthorhombic, space group $P 2{ }_{1} 2_{1} 2_{1}$ (Nr. 19), Stoe IPDS (Imaging Plate) diffractometer, MoK $\alpha, \lambda=0.71073 \AA$, $T=300 \mathrm{~K}, 2 \theta_{\min }=4.1^{\circ}, 2 \theta_{\max }=48.2^{\circ}, \Delta \Phi=0.9^{\circ}, 26676$ measured reflections $( \pm 13, \pm 16,-$ $30: 31), 7595$ independent and 3110 observed reflections [I $>2 \sigma]$, completenes of data $100 \%, R(\mathrm{I})$ $=0.121$, absorption correction: none, extinction correction: none, refinement: SHELXL-93, $N_{\text {ref }}$ $=7595, N_{\mathrm{par}}=568, R_{\mathrm{gt}}(\mathrm{F})=0.0343, R_{\mathrm{w}}\left(\mathrm{F}^{2}\right)=0.0652\left[\mathrm{w}=1 / \sigma^{2}\left(\mathrm{~F}_{0}{ }^{2}\right)\right], \mathrm{S}=0.54, \min . / \max$. residiual electron density $-0.20 / 0.19 \mathrm{e} \AA^{-3}$.

Tetracarbonyl[(R)-1,1'-binaphthalen-2,2'-diyl[bis(di-p-tolylphosphino)]]chromium(0) [(R)9b]. GP, (R)-2,2'-bis(di-p-tolylphosphino)-1,1'-binaphthyl $[(R)$-tol-BINAP, $(R)-8 \mathbf{b} ; 280 \mathrm{mg}$, $0.41 \mathrm{mmol}]$ and $2(106 \mathrm{mg}, 0.41 \mathrm{mmol})$ in THF $(50 \mathrm{~mL})$, heating at reflux for $30 \mathrm{~min}$. Column chromatography (length $30 \mathrm{~cm}, \varnothing 3 \mathrm{~cm}$; TBME/PE 1:8 to 1:3). Pale yellow crystals $(R)-9 b$ (343 mg, $0.41 \mathrm{mmol}) ; \mathrm{mp} 186{ }^{\circ} \mathrm{C}$ (dec.). $[\alpha]_{D}^{20}-42.6$ (c=0.15, THF). IR (KBr): $\widetilde{v} 3048(\mathrm{w})$, 3020 (w), 2960 (w), 2920 (w), 2864 (w), 2000 (s, CO), 1908 (s, CO), 1880 (s, CO), 1596 (w), 1496 (m), 1448 (w), 1396 (w), 1304 (w), 1188 (w), 1088 (m), 1016 (w), 868 (w), 804 (m), 744 (m), $676(\mathrm{~m}), 652(\mathrm{~s}), 580(\mathrm{~m}), 520(\mathrm{~m}) \mathrm{cm}^{-1} .{ }^{1} \mathrm{H}-\mathrm{NMR}\left(400.1 \mathrm{MHz}, \mathrm{THF}-d_{8}\right): \delta 1.92(\mathrm{~s}, \mathrm{br}, 6 \mathrm{H}$, 17-H or 24-H), 2.32 (s, br, 6H, 17-H or 24-H), $6.39(\mathrm{~m}, 4 \mathrm{H}), 6.80(\mathrm{~m}, 2 \mathrm{H}), 7.03(\mathrm{~m}, 2 \mathrm{H}), 7.13$ $(\mathrm{m}, 8 \mathrm{H}), 7.26(\mathrm{~m}, 2 \mathrm{H}), 7.58(\mathrm{~m}, 10 \mathrm{H}) .{ }^{13} \mathrm{C}-\mathrm{NMR}\left(100.6 \mathrm{MHz}\right.$, benzene-d $d_{6}$ APT): $\delta 20.8(-, \mathrm{s}, \mathrm{C}-$ 17 or C-25), $20.9\left(-\right.$, s, C-17 or C-24), $125.7\left(-\right.$, s), $126.1(-, \mathrm{s}), 127.9(+, \mathrm{s}), 128.1\left(-, \mathrm{t}, J_{\mathrm{C}, \mathrm{P}}=\right.$ $5.0 \mathrm{~Hz}), 128.4(+, \mathrm{s}), 128.7(-, \mathrm{s}), 129.4\left(-, \mathrm{t}, J_{\mathrm{C}, \mathrm{P}}=4.5 \mathrm{~Hz}\right), 133.7(+, \mathrm{s}), 133.8\left(-, \mathrm{t}, J_{\mathrm{C}, \mathrm{P}}=5.2\right.$ $\mathrm{Hz}), 134.7\left(-, \mathrm{t}, J_{\mathrm{C}, \mathrm{P}}=6.0 \mathrm{~Hz}\right), 138.4(+, \mathrm{s}), 138.5(+, \mathrm{s}), 139.7(+, \mathrm{s}), 224.1\left(+, \mathrm{CO}_{\mathrm{ax}}, \mathrm{s}\right), 227.3$ $\left(+, \mathrm{CO}_{\text {äq }}, \mathrm{s}\right) .{ }^{31} \mathrm{P}\left\{{ }^{1} \mathrm{H}\right\}-\mathrm{NMR}\left(161.9 \mathrm{MHz}\right.$, benzene- $\left.d_{6}\right): \delta 54.3$ (s). MS-FAB: $\mathrm{m} / \mathrm{z}(\%) 844$ (8) $\left[\mathrm{M}^{+}\right], 816(10)[\mathrm{M}-\mathrm{CO}], 787$ (20) [M-2CO], 786 (50) [M-2CO-1], 759 (75) [M-3CO], 730 
(100) [M-4CO-1]. Anal. Calcd for $\mathrm{C}_{52} \mathrm{H}_{40} \mathrm{CrO}_{4} \mathrm{P}_{2}$ (843.83): C, 74.14; H, 4.78. Found: C, 72.20; H 5.24.

\section{Acknowledgments}

This work was kindly supported by the Deutsche Forschungsgemeinschaft (DFG) and the "Innovationsoffensive Biologisch aktive Naturstoffe: Synthesische Diversität".

\section{References and Notes}

1. Beller, M. Bolm, C., Eds.; Transition Metals for Organic Synthesis; Wiley-VCH: Weinheim, 1998; Vols. 1 and 2.

2. Cornils, B.; Herrmann, W. A., Eds.; Applied Homogeneous Catalysis with Organometallic Compounds; VCH: Weinheim, 1996; Vol. 1 and 2.

3. Catalytic Asymmetric Synthesis; Ojima, I. Ed.; VCH: New York, 1993.

4. Werner, H.; Prinz, R. Z. Naturforsch. B 1966, 22b, 260.

5. Werner, H.; Prinz, R. Chem. Ber. 1967, 100, 265.

6. Chatt, J.; Watson, H. R. J. Chem. Soc. 1961, 4980.

7. Werner, H.; Prinz, R.; Bundschuh, E.; Deckelmann, K. Angew. Chem. 1966, 78, 646. Angew. Chem., Int. Ed. 1966, 5, 606.

8. VanAtta, S. L.; Duclos, B. A.; Green, D. B. Organometallics 2000, 19, 2397.

9. Tekkaya, A.; Özkar, S. J. Organomet. Chem. 1999, 590, 208.

10. Sandhu, S. S.; Mehta, A. K. J. Organomet. Chem. 1974, 77, 45.

11. Andrews, G. T.; Colquhoun, I. J.; McFarlane, W. Polyhedron 1983, 2, 783.

12. Gan, K.-S.; Hor T. S. A. In Ferrocenes: Homogeneous Catalysis. Organic Synthesis, Materials Science; A. Togni, T. Hayashi, Ed.; VCH: Weinheim, 1995; p 3.

13. Rudie, A. W.; Lichtenberg, D. W.; Katcher, M. L.; Davison, A. Inorg. Chem. 1978, 17, 2859.

14. Hor, T. S. A.; Phang, L.-T. J. Organomet. Chem. 1989, 373, 319.

15. Butler, I. R.; Cullen, W. R.; Kim, T.-J.; Rettig, S. J.; Trotter, J. Organometallics 1985, 4, 972.

16. Kagan, H. B.; Dang, T.-P. J. Am. Chem. Soc. 1972, 94, 6429.

17. Whitesell, J. K. Chem. Rev. 1989, 89, 1581.

18. Miyashita, A.; Yasuda, A.; Takaya, H.; Toriumi, K.; Ito, T.; Souchi, T.; Noyori, R. J. Am. Chem. Soc. 1980, 102, 7932.

19. Miyashita, A.; Takaya, H.; Souchi, T.; Noyori, R. Tetrahedron 1984, 40, 1245.

20. Noyori, R.; Takaya, H. Acc. Chem. Res. 1990, 23, 345.

21. Leglaye, P.; Donnadieu, B.; Brunet, J.-J.; Chauvin, R. Tetrahedron Lett. 1998, 39, 9179. 
22. Günther, H. NMR-Spektroskopie; $2^{\text {nd }}$ Edn; Thieme: Stuttgart, 1983.

23. Kalinowski, H.-O.; Berger, S.; Braun, S. 13C-NMR-Spektroskopie; Thieme: Stuttgart, 1984.

24. Still, W. C.; Kahn, M.; Mitra, A. J. Org. Chem. 1978, 43, 2923.

25. Gäbelein, H.; Ellermann, J. J. Organomet. Chem. 1978, 156, 398.

26. Iggo, J. A.; Shaw, B. L. J. Chem. Soc., Dalton Trans. 1985, 1009.

27. Bishop, J. J.; Davison, A.; Katcher, M. L.; Lichtenberg, D. W.; Merrill, R. E.; Smart, J. C. J. Organomet. Chem. 1971, 27, 241.

28. The crystallographic data (without structural factors) of the structures described in this publication were deposited as "supplementary publications no. CCDC 160142 and CCDC 160143" at the Cambridge Crystallography Data Centre. Copies of the data can be obtained with no charge from the following address in Great Britain: The Director, CCDC, 160112 Union Road, Cambridge CB160142 160141EZ (Telefax Int. + 161223/160336-160033; Email: deposit@ccdc.cam.ac.uk). 